\title{
Affirming the Bott Index
}

\section{A useful metric for characterizing the topological behavior of fermions can be extended to bosonic systems as well.}

By Jessica Thomas

W hen physicists began studying topological materials in the 1970s and 1980s, their focus was on the unusual behavior of electrons in certain solids. In these solids, electrons circulate along the surface or edge of an otherwise insulating material. Analogous behavior has now been created in the lab with light, spin waves, and vibrational waves, but it remains an open question whether these bosonic systems can be described by the same topological metrics that characterize electrons, which are fermions. Xiansi Wang and colleagues of the Norwegian University of Science and Technology (NTNU) now use a theoretical model to confirm the viability of a useful metric, known as the Bott index [1].

The researchers consider an infinitely large honeycomb lattice, in which each point has a spin that interacts with its neighbors. They then figure out which collective spin states, or magnons, can form. By design, the chosen spin interactions produce topological behavior-meaning there are robust magnon states along the lattice edges. But Wang and colleagues also ask what happens as they "shrink" the lattice to a finite size. The Bott index has been shown to characterize the onset of topological behavior in a truncated lattice of electrons; the NTNU team

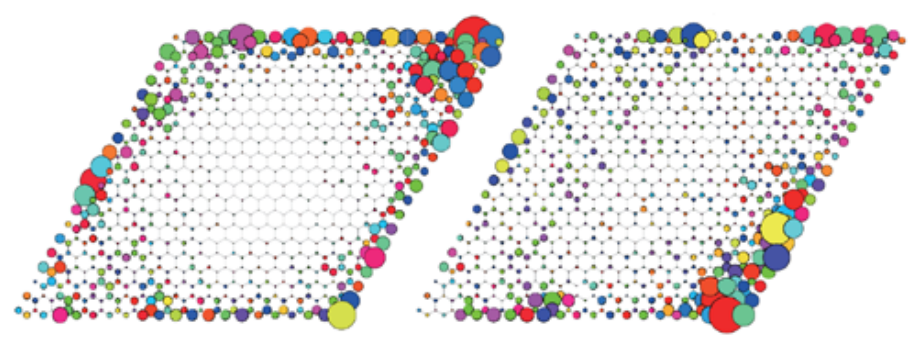

shows that the index works similarly well for bosonic magnons.

Validating the applicability of the Bott index to bosons might help in future studies of topological systems based on light or phonons. But the new study also reveals interesting features specific to magnons. For instance, Wang and colleagues find that, for certain parameters, their model magnons form a state that is similar to the so-called topological Anderson insulator, an electronic phase where the edge states depend on the amount of disorder.

Jessica Thomas is the Editor of Physics.

\section{REFERENCES}

1. X. S. Wang et al., "Bosonic Bott index and disorder-induced topological transitions of magnons," Phys. Rev. Lett. 125, $217202(2020)$.

Credit: X. Wang et al. [1] 